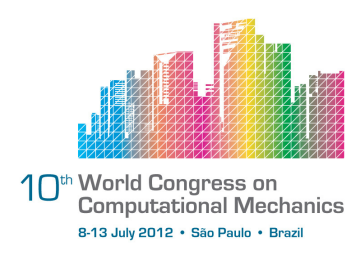

\title{
NUMERICAL SIMULATION OF PARTICULATE EROSION IN TWO-STAGE COMPRESSOR
}

\author{
M. Suzuki ${ }^{1}$, M. Yamamoto ${ }^{2}$ \\ ${ }^{1}$ Aviation Program Group, Japan Aerospace Exploration Agency \\ ${ }^{2}$ Department of Mechanical Engineering, Tokyo University of Science \\ (yamamoto@rs.kagu.tus.ac.jp)
}

\begin{abstract}
When a fluid machine operates in particulate environments, particles suspending in the atmosphere cause the fluid machine some problem. This research is to focus on particulate erosion phenomenon whereby solid particles impinging on a wall cause serious mechanical damage to the wall surface. Particulate erosion leads to unwanted degradation of the performance and life of various machines. Particulate erosion of jet engines is important problem for aviation engineer. Extensive particle ingestion by jet engines occurs at takeoff and landing, which is the principal cause of particulate erosion. The authors investigated performance deterioration in single stage compressor due to particulate erosion, and clarified the mechanism. However, particulate erosion phenomenon in multi stage compressor is not well known although compressor of jet engine consists of multiple stages. In the present study, particulate erosion phenomenon in a two-stage compressor is investigated. The results show that inlet particle velocity of second stage is smaller than that of first stage and the particles cluster around the tip.
\end{abstract}

Keywords: Erosion, Turbomachinery, Computational fluid dynamics.

\section{INTRODUCTION}

In April 2010, volcanic eruption occurred in the southern part of Iceland, and the most of the European airport stopped their service. The event is fresh in our memory. Even if volcanic eruption settles down, volcanic ashes remain in the atmosphere. The volcanic ash particles influence aircrafts and weather conditions over a long period. The particles suspending in the atmosphere cause system troubles and airplane accidents due to the jet engines which operate in such particulate environments. It is not only volcanic ash but also yellow sand (originates in the deserts of East Asia), rain, and ice. Ingestions of these suspending particles to jet engines leads to some problems, e.g., erosion, deposition, and icing. Hence the latest research in these phenomena shows more interest in protecting industrial machines.

Particulate erosion is a phenomenon whereby solid particles suspending in the gas flow, e.g., volcanic ash and yellow sand, impinge on a wall, and cause mechanical damage to 
the wall surface. The phenomenon involves a typical two-phase, gas-particle turbulent flow and can be considered to be a multi-physics problem in which the flow field, particle trajectory and wall deformation interact. Particulate erosion leads unwanted degradation of the performance and life of various machines. Furthermore, severe particulate erosion causes miserable accident. Thus, for safe operation, requirements of type approval (aviation certification) include requirements that jet engines are able to operate without trouble after sand ingestion.

Extensive particle ingestion by jet engines occurs at takeoff and landing, which is the principal cause of particulate erosion. In particular, the fan and compressor of aircraft engines are subjected to severe damage. In general turbofan engines, the rotor blades, stator vanes, and the shroud are damaged. Thus, these eroded components must be repaired and exchanged after every approximately $10,000 \mathrm{~h}$ of operation. Since the interval depends on the airfoil geometry, the axial location, the root structure, and the operation cycle, the design requires consideration of particulate erosion. Although experimental measurements give useful data, expensive facilities and lengthy testing are required for the experiments. Moreover, the testing is destructive. If particulate erosion can be numerically predicted in the development/design phase, the number of prototypes can be reduced. Furthermore, this prediction can increase product lifetime, prevent performance deterioration, and reduce the frequency of overhauls.

In recent years, numerical simulations based on computational fluid dynamics (CFD) have been performed, e.g., [1-3]. However, in these simulations, changes in the flow field and the related particle trajectories during the erosion process were not taken into account. This treatment is physically unrealistic since they consider only one-way effects from the continuous-phase to the dispersed-phase and from the particle trajectories to wall deformation. Consequently, surface erosion over time cannot be reproduced precisely. However, this procedure can predict short-time erosion, and is useful tool for initial design phase.

As a step forward, developed herein is a numerical procedure to investigate particulate erosion, including the temporal change in the two-phase flow field related to changes in the wall shape. The proposed procedure can reproduce any wall deformation by resolving the wall shape using computational grids. In addition, the interactions can be simulated by repeating a cycle that consists of three steps: the continuous phase, the dispersed phase, and the wall deformation due to particulate erosion. This concept is applicable to any particulate erosion phenomenon. The appropriate equations for the continuous phase, the dispersed phase, and the wall deformation can be selected according to the computational objective. This numerical code was successfully applied to some particulate erosion problems, e.g., a 90-degree bend [4], a turbine vane [5] and a single stage axial flow compressor [6, 7].

The performance deterioration in single stage compressor due to particulate erosion have been investigated and clarified the mechanism [6, 7]. However, particulate erosion phenomenon in multi stage compressor is not well known although compressor of jet engine consists of multiple stages. In the present study, particulate erosion phenomenon in a two-stage compressor is investigated. The investigation focuses on the influence of upstream stage on downstream stage. Two cases are calculated with different particle diameters: 5 and $165 \mu \mathrm{m}$. 


\section{NUMERICAL PROCEDURE}

\subsection{Outline}

It is assumed herein that the particle concentration in the flow field is small enough to ignore particle-particle collisions and particle-phase interactions with the flow field. Additionally, as particulate erosion occurs over long time periods, the changes in the flow field can be regarded as quasi-steady state. Therefore, steady-state or time-averaged flow distributions are employed for each eroded geometry at every instance. Consequently, in the present study, particulate erosion is approximated to a series of quasi-steady states.

The computational procedures for prediction of particulate erosion are: (1) calculate the turbulent flow field, (2) calculate the particle trajectories, and (3) change the wall shape. These procedures are repeated iteratively until the computational time reaches the prescribed termination time. A more detailed description can be found in [4].

\subsection{Gas-phase}

Numerical simulations are conducted using a finite difference technique. The gasphase is considered to be a continuous phase, while the particle-phase is dispersed. As mentioned above, the particle-phase has no influence on the gas-phase (i.e., one-way coupling). The gas-phase flow is assumed to be three-dimensional, compressible and turbulent. It is calculated using the Eulerian approach, based on the Favre-averaged continuity equation, Navier-Stokes equations and energy equation (i.e., RANS approach).

$$
\begin{gathered}
\frac{\partial \bar{\rho}}{\partial t}+\frac{\partial}{\partial x_{j}}\left(\overline{\rho \tilde{u}_{j}}\right)=0 . \\
\frac{\partial}{\partial t}\left(\bar{\rho}_{u_{i}}\right)+\frac{\partial}{\partial x_{j}}\left\{\bar{\rho}_{u_{i}} \widetilde{u}_{j}+\bar{p} \delta_{i j}-\left(\bar{\tau}_{i j}-\overline{\rho u_{i}^{\prime \prime} u_{j}^{\prime \prime}}\right)\right\}=\tilde{\rho f}_{i} . \\
\frac{\partial}{\partial t}\left(\bar{\rho}_{t}\right)+\frac{\partial}{\partial x_{j}}\left\{\left(\bar{\rho}_{t}+\bar{p}\right) \widetilde{u}_{j}-\left(\bar{\tau}_{i j} \widetilde{u}_{i}-\bar{q}_{j}-\overline{\rho e_{t}^{\prime \prime} u_{j}^{\prime \prime}}\right)\right\}=\tilde{\rho f}_{j} \widetilde{u}_{j} .
\end{gathered}
$$

where: $x_{i}$ is the location in Cartesian coordinates; $u_{i}, f_{i}, q_{i}$, and $\tau_{i j}$ are the velocity, external force, heat flux and viscous stress tensor, respectively; $t, \rho, p$, and $e_{t}$ denote the time, density, static pressure, and total energy of the fluid, respectively; $(-),(\sim)$, and (")denote the Reynolds averaging operation, the Favre averaging operation, and the fluctuating component, respectively; in the turbulence model, the standard $k-\varepsilon$ model is used (Launder and Spalding, [8]); Coriolis and centrifugal forces are considered to be external.

The governing equations are discretized using the second-order upwind TVD scheme (Yee and Harten, [9]) for the inviscid terms, the second-order central difference scheme for the viscous terms, and the four-stage Runge-Kutta method (Jameson and Baker, [10]) for the time integration. In order to reduce the time required to obtain the steady state solutions, the local time stepping method is employed. 


\subsection{Particle-phase}

For the particle-phase, the Lagrangian approach is used to track particles over time along their trajectories through the flow field. The particles are assume to be spherical and non-rotating. The strictest momentum equation of spherical particles is the B-B-O equation [11]. However, as mentioned above, particle-particle collisions and particle-phase interactions within the flow field are neglected. The buoyancy force, the pressure gradient force, the added mass force, and the Basset history force are also negligible because the difference between the density of the solid particles and the fluid is large and the free-stream velocity is high. For these assumptions, the equations of motion for the particles are:

$$
\begin{gathered}
\frac{d x_{p, i}}{d t}=u_{p, i} \\
\frac{d u_{p, i}}{d t}=f_{D, i}+f_{I, i}
\end{gathered}
$$

where: $p$ denotes the particle; and $f_{D, i}$ and $f_{I, i}$ are the drag force and inertia force, i.e. the Coriolis and centrifugal force, respectively.

When the particle impinges on the wall, the particle velocity after impact decreases. This decrease depends on the kinetic energy consumed by erosion. More specifically, the velocity components corresponding to the energy consumed by deformation and cutting wear are subtracted from the vertical and horizontal velocity components relative to the wall surface.

\subsection{Erosion estimation}

To estimate the degree of erosion, the model proposed by Neilson and Gilchrist [12] is applied. This model considers damage due to particle impacts to be due to two separable mechanisms: deformation wear caused by the normal velocity and cutting wear due to the tangential velocity. Each component has associated wear, $W_{d}$ and $W_{c}$, respectively. The total weight loss $W_{t}$ is the sum of these:

$$
\begin{gathered}
W_{t}=W_{d}+W_{c} \\
W_{d}=\frac{\frac{1}{2} M(V \sin \alpha-K)^{2}}{\psi} \\
W_{c}=\left\{\begin{array}{cc}
\frac{1}{2} M V^{2} \cos ^{2} \alpha \sin n \alpha \\
\phi & \left(\alpha<\alpha_{0}\right) \\
\frac{\frac{1}{2} M V^{2} \cos ^{2} \alpha}{\phi} & \left(\alpha \geq \alpha_{0}\right) \\
\alpha_{0}=\frac{\pi}{2 n} &
\end{array}\right.
\end{gathered}
$$

where: $M$ is the total mass of the particles; $\alpha$ and $V$ are the attack angle and impinging velocity of a particle, respectively; $K$ is the normal velocity's threshold value, below which no de- 
formation wear occurs; $\alpha_{0}$ is the attack angle at which the tangential component of the reflection velocity becomes zero; and $\psi$ and $\phi$ represent the energy needed to remove a unit weight of material from the wall by deformation and cutting wear, respectively.

\section{COMPUTATIONAL CONDITIONS}

The computations are carried out for the single-stage axial flow compressor measured by Balan and Tabakoff [13]. The compressor does not have any guide vanes. The compressor design is almost two-dimensional. The hub diameter and the tip diameter are constant in the axial direction, and the airfoil and stagger angle are constant in the spanwise direction. The computational conditions are set to be identical to those in the experiments. The wheel speed and the mass flow rate are fixed to $5,000 \mathrm{rpm}$ and $1.73 \mathrm{~kg} / \mathrm{s}$, respectively. In order to estimate the compressor performance, the compressor is operated at various mass flow rates without sand ingestion.

The computational domain is based on the multi-block method and the overset grid method and consists of six parts: the inlet, the main grid of the rotor, the sub-grid of the rotor, the main grid of the stator, the sub-grid of the stator, and the outlet (see Fig. 1). The sub-grids around the rotor blade and the stator vane are C-type sub-grids. The number of grid cells in the flow fields is approximately 430,000, and the number of grid cells within the wall regions is approximately 660,000. Thus, the total number of grid cells is approximately $1,090,000$. Note that this grid is applied based on grid independence tests using coarser and finer grids. The regions around the rotor blade are computed in a rotating coordinate system, and the other regions are treated in a stationary coordinate system.

The boundary conditions are imposed as follows. At the inlet boundary, the Mach number is extrapolated from the downstream region. The flow angle, the total pressure, and the total temperature are fixed. At the exit, the static pressure is fixed and the other variables are extrapolated from the upstream region. On the wall surfaces, adiabatic and slip conditions

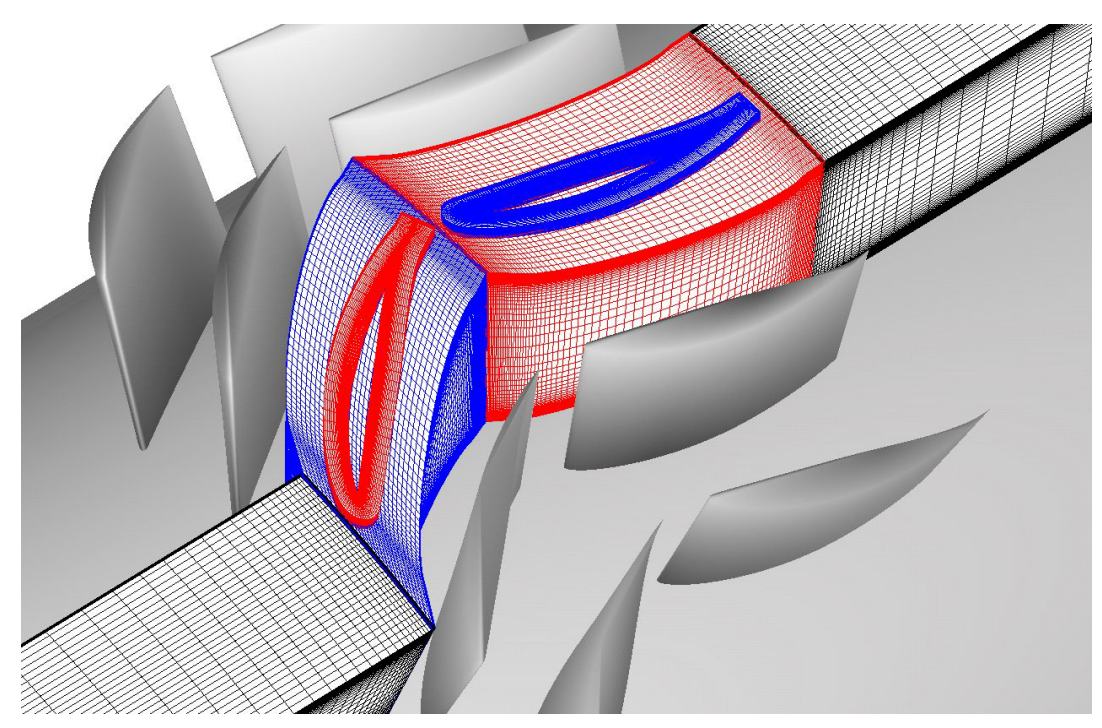

Figure 1. Computational grid 
with the wall function are imposed. At the circumferential boundaries, the periodic condition is used and only 1 pitch is computed for each cascade because the number of rotor blades is the same as the number of stator vanes. The changes in the relative locations of the rotor blade and the stator vane are consistently considered by that the calculation of interpolation parameters for rotor and stator interface is conducted at every instance of time integration.

In the experiment by Balan and Tabakoff [13], the compressor was eroded in steps using $5 \mathrm{~kg}$ of sand during each step. The duration of sand ingestion for each step was $121 \mathrm{~s}$. The erosion test was conducted in 5 steps, and so the total mass of ingested sand was $25 \mathrm{~kg}$ over $605 \mathrm{~s}$. In the simulations, a cycle consisting of gas-phase, particle-phase, and wall-shape calculations is carried out for 1 step of the experiment, in which 5 cycles are performed. The walls are constructed of aluminum (density: $2.70 \times 10^{3} \mathrm{~kg} / \mathrm{m}^{3}$ ), and the solid particles are quartz (density: $2.65 \times 10^{3} \mathrm{~kg} / \mathrm{m}^{3}$ ). In order to determine the erosion parameters, experimental data reported by Grant and Tabakoff [14] on collisions between quartz and aluminum are used. The fitted values are $\psi=24.0 \times 10^{8} \mathrm{~m}^{2} / \mathrm{s}^{2}, \phi=8.0 \times 10^{8} \mathrm{~m}^{2} / \mathrm{s}^{2}, n=4.5$, and $K=1.2 \mathrm{~m} / \mathrm{s}$. Two cases are calculated with different particle diameters: 5 and $165 \mu \mathrm{m}$.

In the present study, particulate erosion phenomenon in a two-stage compressor is investigated. It is difficult to simulate directly multi-stage compressor since the numerical cost is high. To reduce the numerical cost, it is assumed that the gas flow field in second stage is same as that in first stage. Thus, the gas-phase is computed for only one stage. On the other hand, the particle-phase and erosion in the first sage and second stage are calculated with different inlet conditions on the particle velocity and position. The inlet condition of second stage is given from the outlet results of the first stage.

\section{RESULTS AND DISCUSSION}

\subsection{Gas-phase}

Figure 2 shows the streamlines in the rotor and stator passages at peak efficiency before erosion. The color corresponds to the velocity. The results for the rotor passage are shown in a relative coordinate system, and results for the stator passage are exhibited in an absolute coordinate system. The compressor is a subsonic compressor. The inlet Mach number is less than 0.2, and the maximum value of the local Mach number is less than 0.3. A leakage vortex is confirmed to exist at the tip of the rotor blade. Although the instantaneous results are presented herein, the regions around the trailing edge of the rotor blade and the tip leakage vortex exhibit unsteadiness, while the other regions of the rotor passage are approximately steady. The flow field in the stator passage changes periodically owing to the influence of the rotor blade wake.

\subsection{Particle-phase}

Figure 3 illustrates a typical particle trajectory for particle diameter of $165 \mu \mathrm{m}$ in first stage. The particle velocity is represented by color. The particle trajectory from approximately 


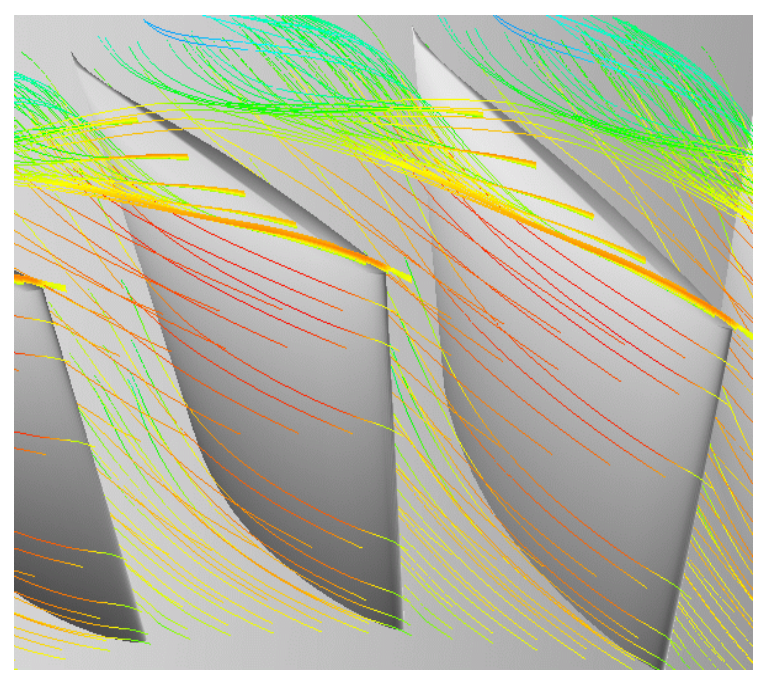

(a) Rotor passage

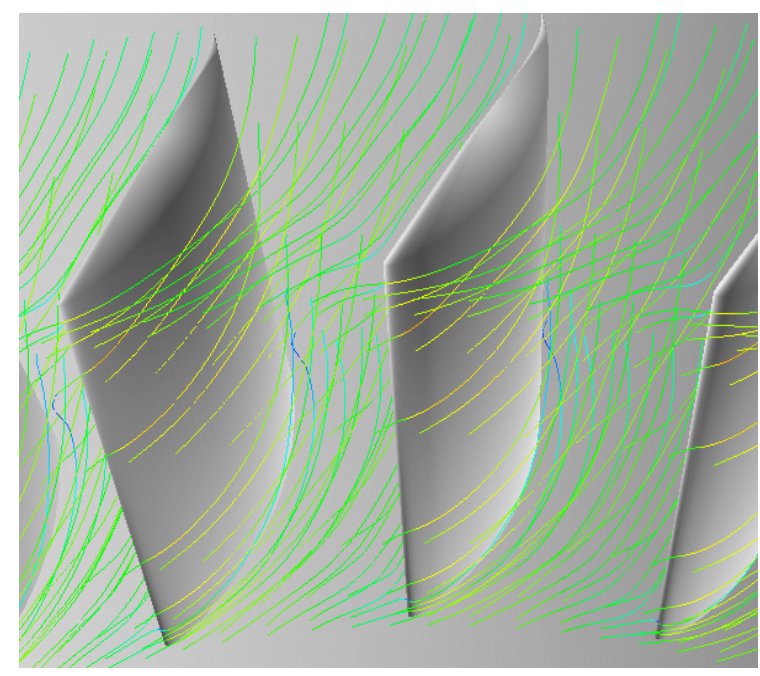

(b) Stator passage

0 $150[\mathrm{~m} / \mathrm{s}]$

Figure 2. Streamlines at peak efficiency before erosion

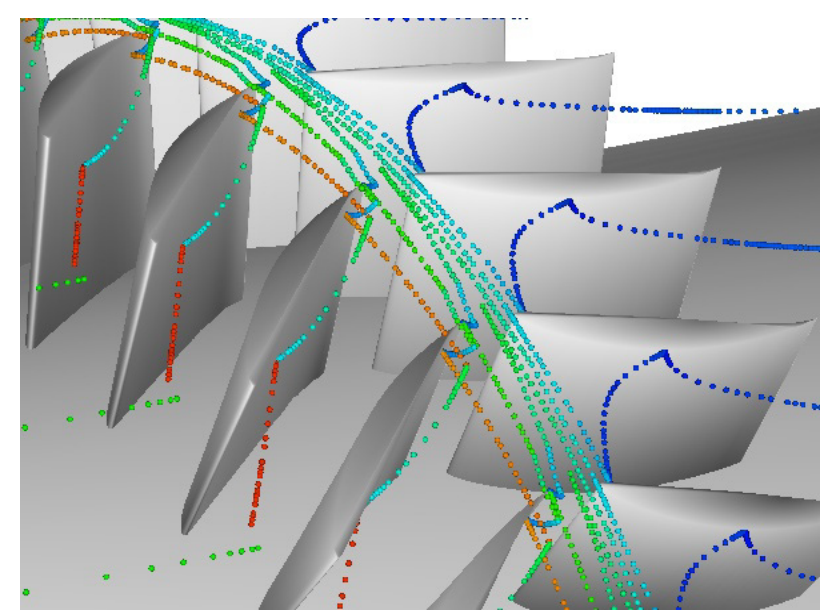

0

$90[\mathrm{~m} / \mathrm{s}]$

Figure 3. Typical particle trajectory

0.1 of the chord length upstream of the leading edge of the rotor blade to approximately 0.1 of the chord length downstream of the trailing edge of the rotor blade is shown in a relative coordinate system. The reason the trajectory does not appear to be continuous is that the trajectory is exhibited in the relative coordinate system around the rotor blade.

Most of the particles impact the leading edge and the pressure side of the rotor blade. In Fig. 3, the particle impinges on the pressure surface of the rotor blade, and most of the kinetic energy is consumed by the first impact. After the particle-wall collision, the particle velocity is reduced, and the particle then moves toward the tip of the rotor blade due to centrifugal force. At this time, the relative velocity with respect to the outer casing is approximately equal to the rotational speed of the rotor because the particle has a low circumferential veloc- 
ity. The particle impacts the outer casing, damages the casing surface, and accelerates after the particle-casing impingement. The accelerating particle moves downstream within the tip clearance along with repeated impacts on the blade tip and the outer casing and passes through the rotor passage.

When a particle arrives at the stator passage, the particle has a low axial velocity. The impinging velocity on the stator vane is determined based on the circumferential velocity of the particle at the exit of the rotor passage and rotor wheel speed. Although the particle impinges repeatedly on the stator vane and the casing, the first impact is the primary reason of the erosion of the stator vane owing to the high impinging velocity. Under this condition, the particle diameter is fairly large. In addition, the particle acceleration is extremely slow, and so the impinging velocity on the stator vane is low during and after secondary impact. On the other hand, a marked erosion characteristic is observed in rotor-stator interaction, namely, particles impinge on the leading edge of the stator vane, and the rebounding particles impact the trailing edge of the rotor blade and cause erosion.

Figure 4 plots particle trajectories in axial-radial section for particle diameters of 5 and $165 \mu \mathrm{m}$ in first stage and second stage. Inlet particle velocity of second stage is smaller than that of first stage due to particle-wall impingements, and so the particles in second stage impinge on near the leading edge. This leads to increased damage of the leading edge. As mentioned above, the particles cluster around the tip by centrifugal force. This tendency is clearer in second stage than in first stage, since the low speed particles are significantly affected by

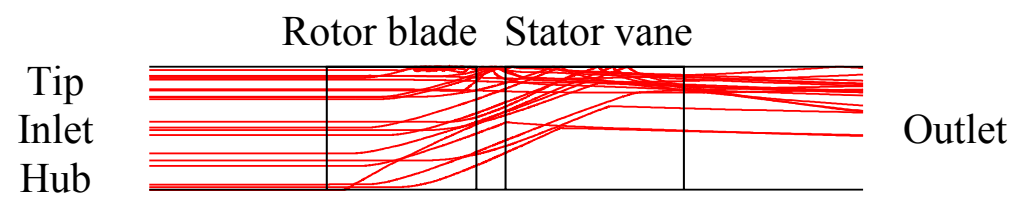

(a) 1st stage, $5 \mu \mathrm{m}$

Tip

Rotor blade Stator vane

Inlet

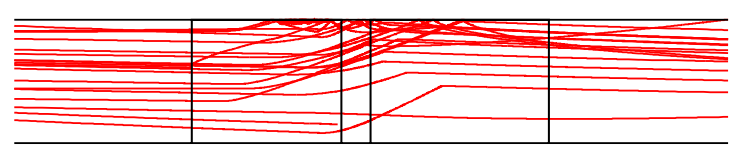

Outlet

(b) 2nd stage, $5 \mu \mathrm{m}$

Tip

Rotor blade Stator vane

Inlet

Hub

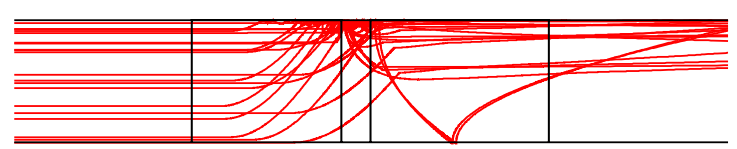

Outlet

(c) 1st stage, $165 \mu \mathrm{m}$

Tip

Rotor blade Stator vane

Inlet

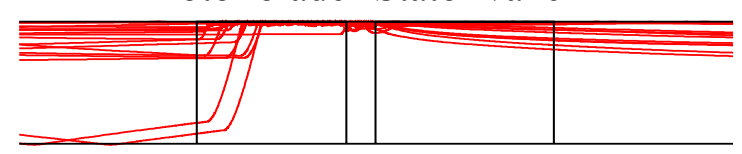

Outlet

(d) 2nd stage, $165 \mu \mathrm{m}$

Figure 4. Particle trajectories in axial-radial section 
centrifugal force. On the other hand, the smaller particle diameter is, the stronger influence of the gas-phase flow on the particles becomes. Because the outflow velocity of the gas-phase is almost equal to the inflow velocity of the gas-phase, if the gas-phase flow strongly affects on the particles, the outflow velocity of the particles approaches the inflow velocity of the particles. Since the small particles easily follow gas-phase flow, the lost kinetic energy by the particle-wall impingements recovers, and so the inertia of the particles is more dominant than the centrifugal force. Therefore, spanwise motion of the small particles is not significant and the particle concentration of the small particles at the tip is lower than that of the large particles.

\subsection{Erosion}

Erosion depth of rotor blade and stator vane in first stage is illustrated in Fig. 5. The pressure surface, suction surface, leading edge and trailing edge, respectively, are denoted as P.S., S.S., L.E. and T.E. in Fig. 5.

The pressure surface of the rotor blade suffers over the entire surface area (see Fig. 5 a). The leading edge and the tip are severely damaged. Since the solid particles coming from the inlet directly impacts the pressure side of the rotor blade, a wide area suffers from erosion, so that the surface becomes very rough. The leading edge and the tip of the rotor blade suffer severe damage because the frequency of particle impingement is higher than in other regions. Since the particles flow along the camber of the blade, the projection area of the leading edge is large, and the particles tend to concentrate at the leading edge. The severe erosion of the tip results from the particles propelled by centrifugal force toward the tip repeatedly impinge on the tip and the outer casing, as shown in Fig. 3 and 4, and the inflow particles directly impinge on the tip.

On the other hand, the suction side of the rotor blade is not eroded, except for the leading edge (see Fig. 5 b). This is explained as follows. The particles rebounding from the pres-

L.E.

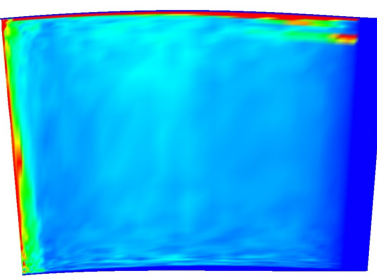

(a) Rotor blade P.S.

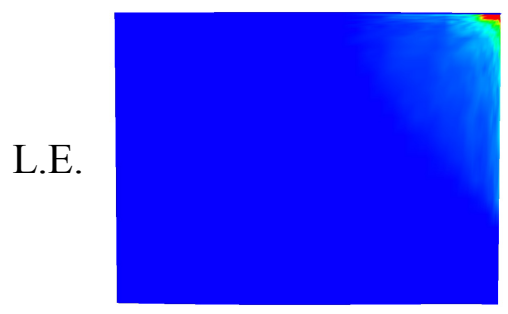

(c) Stator vane P.S.

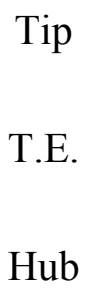

Tip
T.E.
Hub

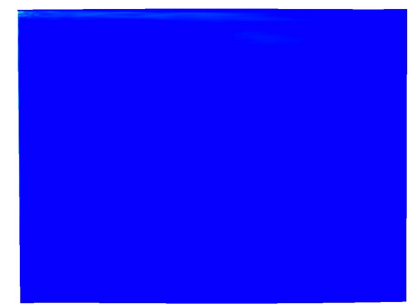

(d) Stator vane S.S.
L.E. $\quad 1.0[\mathrm{~mm}]$

L.E.

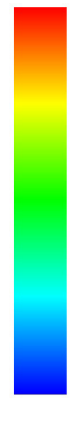

0.0

Figure 5. Erosion depth of rotor blade and stator vane in first stage 
sure side never reach the suction surface and impinge on the outer casing, because the particles have consumed most of the kinetic energy due to erosion. The particles then move toward the pressure side.

On the pressure surface of the stator vane (see Fig. 5 c), the eroded region is concentrated at the tip of the stator vane near the leading edge. The leading edge is severely damaged because the impact frequency is high near the leading edge. This is because the axial velocity of the particles is low due to the impingement on the rotor blade and the casing. The tip of the stator vane suffers severe erosion because of the cluster of particles on the side of the tip of the stator vane caused by the centrifugal force after impingement in the rotor passage.

In contrast to the suction surface of the rotor blade, slight erosion is observed on the suction surface near the tip of the stator vane (see Fig. 5 d). Some particles rebounding from the pressure surface of the stator vane impinge on the suction surface. This is attributed to the particles rebounding from the pressure surface of the stator vane having low axial velocity and the recovery of the axial velocity being slow because of the large particle diameter. However, since the impinging velocity is fairly low, the erosion damage is slight.

Figure 6 depicts erosion depth of rotor blade and stator vane in second stage. Tendencies of first stage is emphasized in second stage. Eroded region in second stage becomes narrower than first stage. In addition, maximum erosion depth in second stage is deeper than that in first stage, namely, erosion damage of the leading edge and the tip of second stage are severer than fist stage.

\section{CONCLUSIONS}

Numerical simulation of particlate erosion in a two-stage compressor is performed in order to investigate influence of upstream stage on downstream stage. Through the present study, the following insights are obtained:

L.E.

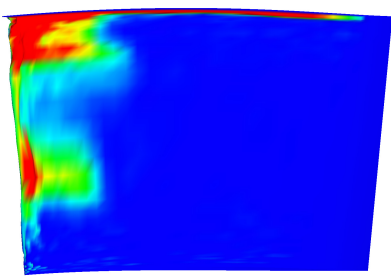

(a) Rotor blade P.S.

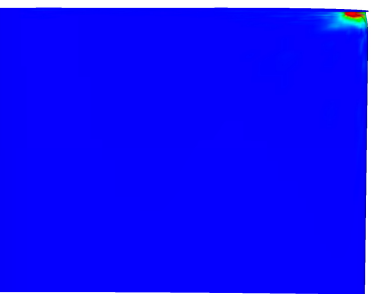

(c) Stator vane P.S.

\section{Tip \\ T.E. \\ $\mathrm{Hub}$}

(b) Rotor blade S.S.

Tip
T.E.
Hub

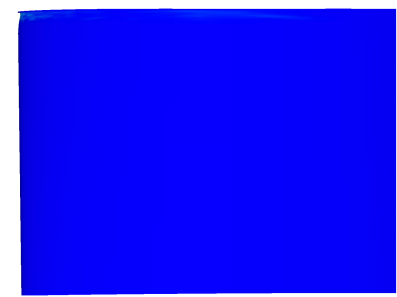

(d) Stator vane S.S.
L.E. $\quad 1.0[\mathrm{~mm}]$

L.E.

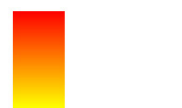

0.0

Figure 6. Erosion depth of rotor blade and stator vane in second stage 
(1) Most of the particles impact the leading edge and the pressure side of the rotor blade, and this is the primary cause of the rotor blade damage.

(2) When particle-wall collision reduces the particle velocity, centrifugal force moves the particles towards the tip.

(3) Inlet particle velocity of second stage is smaller than that of first stage due to particle-wall impingements.

(4) The particles cluster around the tip by centrifugal force. This tendency is clearer in second stage than in first stage, since the low speed particles are significantly affected by centrifugal force.

(5) Since the small particles easily follow gas-phase flow, the lost kinetic energy by the particle-wall impingements recovers, and so the difference of first stage and second stage is small.

(6) Eroded region in second stage becomes narrower than first stage and erosion damage of the leading edge and the tip of second stage are severer than first stage.

In the present study, to reduce the numerical cost, it is assumed that the gas flow field in second stage is same as that in first stage. This assumption degrades the accuracy of numerical results. As a step forward, further investigations which computes directly multi-stage compressor will be conducted.

\section{REFERENCES}

[1] Hamed A. A., Tabakoff W., Rivir R. B., Das K., Arora P., "Turbine blade surface deterioration by erosion". Journal of Turbomachinery 127, 445-452, 2005.

[2] Sato S., Shimizu A., Yokomine T., "Numerical prediction of erosion for suspension flow duct". Wear 186-187, 203-209, 1995.

[3] Wang J., Shirazi S. A., "A CFD based correlation for erosion factor for long-radius elbows and bends". J. Energ. Resour. Tech. 125, 26-34, 2003.

[4] Suzuki M., Inaba K., Yamamoto M., "Numerical simulation of sand erosion in a squaresection 90-degree bend". J. Fluid. Sci. Tech. 3, 868-880, 2008.

[5] Suzuki M., Toda K., Yamamoto M., "Numerical simulation of sand erosion phenomena on turbine blade surface". Proc. WCCM VI, 1-10, 2004.

[6] Suzuki M., Inaba K., Yamamoto M., "Numerical simulation of sand erosion phenomena in rotor/stator interaction of compressor". J. Therm. Sci. 17, 125-133, 2008.

[7] Suzuki M., Yamamoto M., "Numerical simulation of sand erosion phenomena in a singlestage axial compressor". J. Fluid. Sci. Tech. 6, 98-113, 2010.

[8] Launder B. E., Spalding D. B., "The numerical computation of turbulent flows", Comput. Meth. Appl. Mech. Eng. 3, 269-289, 1974. 
[9] Yee H. C., "Upwind and symmetric shock-capturing schemes", NASA-TM-89464, 1-127, 1987.

[10] Jameson A., Baker T. J., "Solution of the Euler equations for complex configurations", AIAA-83-1929, 293-302, 1983.

[11] Tchen C., "Mean value and correlation problems connected with the motion of small particles suspended in a turbulent fluid", Ph. D. thesis, Technische Hogeschool, Delft, Holland, 1947.

[12] Neilson J. H., Gilchrist A., "Erosion by a stream of solid particle", Wear 11, 111-122, 1968.

[13] Balan C., Tabakoff W., "Axial flow compressor performance deterioration”, AIAA-841208, 1-13, 1984.

[14] Grant G., Tabakoff W., "Erosion prediction in turbomachinery resulting from environmental solid particles", J. Aircraft 12, 471-478, 1975. 